\title{
Early left ventricular regional contractile impairment in chronic mitral regurgitation occurs in a consistent, heterogeneous pattern
}

\author{
Hersh S. Maniar, MD, Beckah D. Brady, BS, Urvi Lee, PhD, Brian P. Cupps, PhD, Julia Kar, PhD, \\ Kathleen M. Wallace, MSW, and Michael K. Pasque, MD
}

\begin{abstract}
Objectives: The clinical guidelines for asymptomatic patients with chronic mitral regurgitation (MR) use the ejection fraction (EF) to trigger surgical referral. We hypothesized that the EF is not sensitive enough to detect the earliest contractile injury in chronic MR and that the injury associated with chronic MR is not global but heterogeneous, occurring regionally and predictably, before the onset of global left ventricular (LV) dysfunction.

Methods: Fifteen patients with chronic MR and normal LVEF by echocardiography underwent cardiac magnetic resonance imaging with tissue tagging. Point-specific comparisons (at 15,300 LV grid points) of multiple strain parameters to a normal human strain database allowed normalization of patient-specific regional contractile function. Data were mapped over patient-specific 3-dimensional geometry and averaged across $6 \mathrm{LV}$ regions.
\end{abstract}

Results: Global LV longitudinal and circumferential myocardial strains were normal for all 15 patients with MR compared with normal controls $(P>.05)$. Despite preserved global function, the anteroseptum and posteroseptum demonstrated significantly worse contractile function compared with other LV regions $(P=.003$ and $P=.035$, respectively). Hypercontractile regions (lateral walls) appeared to compensate $(P=.002)$ for the reduced septal contractile function, masking injury detection by global indexes.

Conclusions: The earliest contractile injury seen in patients with MR is heterogeneous and consistently distributed along the LV septum. Compensatory responses include hypercontractility of other regions. These data suggest that rather than relying on global LV contractile metrics, which cannot detect early injury, patients might be better served by undergoing directed surveillance of "sentinel" LV regions (LV septum) with high-resolution metrics of regional contractile function. (J Thorac Cardiovasc Surg 2014;148:1694-9)

Degenerative mitral valve disease is the most common valvular heart disease in America. Given its increasing incidence with age, it has been estimated that $>5$ million people will have either moderate or severe mitral regurgitation (MR) by $2030 .^{1}$ Several studies have demonstrated that the presence of even moderate MR is associated with increased mortality, and severe MR, even in asymptomatic patients, has been associated with an increase in all-cause mortality, cardiac mortality, and cardiac morbidity. ${ }^{2}$ Moreover, symptomatic patients and patients with even mildly depressed ventricular function (ejection fraction

From the Department of Surgery, Washington University School of Medicine, St Louis, Mo.

This work was supported by funding from the American Heart Association (grant 11CRP7380050) and partially by funding from the National Institutes of Health (grants HL064869 and HL069967).

Disclosures: Drs Pasque and Cupps and Washington University could receive income based on a license of related technology by the University to CardioWise, LLC. CardioWise, LLC, did not provide funding for the present study. All other authors have nothing to disclose with regard to commercial support.

Received for publication April 10, 2014; revisions received May 8, 2014; accepted for publication May 23, 2014.

Address for reprints: Hersh S. Maniar, MD, Department of Surgery, Washington University School of Medicine, 660 South Euclid Ave, Campus Box 8234, St Louis, MO 63110 (E-mail: maniarh@wustl.edu).

$0022-5223 / \$ 36.00$

Copyright (C) 2014 by The American Association for Thoracic Surgery

http://dx.doi.org/10.1016/j.jtcvs.2014.05.088
$[\mathrm{EF}]<60 \%$ ) have fared much worse, with survival as low as $20 \%$ at 4 years. ${ }^{3}$

Mitral valve surgery remains the standard treatment of degenerative mitral valve disease, and advances in surgical technique have allowed experienced centers to achieve high rates of successful valve repair with low observed morbidity and mortality. ${ }^{4-6}$ The long-term success for patients undergoing mitral valve surgery, however, depends on preservation of LV contractile function. Patients referred for surgery early, before the onset of irreversible contractile injury, have been shown to regain normal life expectancy. Thus, the current American College of Cardiology/American Heart Association guidelines have recommended early referral for mitral valve surgery in patients with minimal symptoms or early signs of ventricular embarrassment if mitral valve repair is likely. ${ }^{6-8}$

Although clinicians have agreed that surgery for MR should be performed before the onset of significant irreversible myocardial injury, the identification of that specific point for a given individual patient has been problematic. Two fundamental issues have contributed to the difficulty of preserving ventricular function in patients with MR: (1) identifying a metric with the ability to identify the earliest signs of ventricular injury and (2) a lack of knowledge of the manner or pattern by which earliest LV contractile injury presents. 


$$
\begin{aligned}
& \text { Abbreviations and Acronyms } \\
& \qquad \begin{aligned}
\mathrm{BNP} & =\mathrm{B} \text {-type natriuretic peptide } \\
\mathrm{EF} & =\text { ejection fraction } \\
\mathrm{LV} & =\text { left ventricular } \\
\mathrm{MR} & =\text { mitral regurgitation } \\
\mathrm{MRI} & =\text { magnetic resonance imaging }
\end{aligned}
\end{aligned}
$$

The purpose of the present investigation was to test the hypothesis that multiparametric strain analysis using magnetic resonance imaging (MRI) and tissue tagging could identify early LV dysfunction in minimally or asymptomatic patients and that the volume loading associated with MR creates a contractile injury that is regionally and heterogeneously distributed in a predictable pattern.

\section{METHODS}

\section{Patient Characteristics}

From August 2010 to July 2013, 15 asymptomatic or minimally symptomatic patients with isolated moderate to severe degenerative MR underwent evaluation of their LV function using transthoracic echocardiography and tagged cardiac MRI. The patient demographics and comorbidities are listed in Table 1. Most patients were New York Heart Association class I, with normal B-type natriuretic peptide (BNP) levels. Of the 15 patients, 3 $(20 \%)$ were taking afterload reduction medications, $4(26 \%)$ were taking $\beta$-blockers, and $2(13 \%)$ were receiving diuretic therapy. No patient had ischemic MR. All patients with any evidence of coronary artery disease were specifically excluded from the study group.

\section{Normal Strain Database}

Sixty healthy volunteers (Table 1) with no known cardiac disease or hypertension underwent similar myocardial strain analysis and contributed complete LV systolic strain information to a normal human strain database.

The Human Research Protection Office at Washington University (St Louis, Mo) approved the present study, and all subjects gave written informed consent. No sex-based or racial/ethnic-based exclusions were used during patient recruitment.

\section{Cardiac MRI}

Electrocardiogram-gated short- and long-axis tagged MRI scans were acquired from end-diastole through systole using a 1.5T scanner (Avanto; Siemens Medical Systems, Malvern, Pa). In each imaging plane, a spatial modulation of magnetization radiofrequency tissue-tagging preparation ${ }^{9}$ was applied, followed by a 2-dimensional balanced steady-state free precession cine image acquisition. Short-axis images covered the entire heart, and long-axis images were acquired in 4 radially oriented planes. The typical imaging parameters were as follows: tag spacing, $8 \mathrm{~mm}$; slice thickness, $8 \mathrm{~mm}$; repetition time, $32.4 \mathrm{~ms}$; echo time, $1.52 \mathrm{~ms}$; field of view, $350 \times 350 \mathrm{~mm}$; and image matrix, $192 \times 256$. In the same breath hold, anatomic and tagged images were acquired at the corresponding slice positions.

\section{Strain Analysis}

Strain measurements were obtained using a previously described and validated method. ${ }^{10,11}$ In brief, endocardial and epicardial wall boundaries were manually segmented on the anatomic MRI scans. These wall boundaries were transferred to the tagged images to process the tagged data. The tag lines were tracked on the images in a semiautomated fashion using an active contour approach. Displacements were calculated
TABLE 1. Characteristics of patients with mitral regurgitation and healthy volunteers

\begin{tabular}{lcc}
\hline \multicolumn{1}{c}{ Characteristic } & Patients with MR & Healthy volunteers \\
\hline Age (y) & $49.3 \pm 11.3$ & $33.1 \pm 10.8$ \\
Female sex & $20(3 / 15)$ & $53(32 / 60)$ \\
Diabetes & 0 & 0 \\
HTN & $13(2 / 15)$ & 0 \\
COPD & 0 & 0 \\
CRI & 0 & 0 \\
Atrial fibrillation & 0 & 0 \\
NYHA I & $67(10 / 15)$ & $100(60 / 60)$ \\
NYHA II & $33(5 / 15)$ & 0 \\
BNP & $34.0 \pm 28.5^{*}$ & NA \\
Echocardiographic ejection & $61.5 \pm 5.8$ & NA \\
$\quad$ fraction (\%) & & \\
Echocardiographic end-systolic & $3.4 \pm 0.4$ & NA \\
$\quad$ dimension (cm) & & NA \\
Echocardiographic left atrial & $4.4 \pm 0.5$ & \\
$\quad$ size (cm) & & NA \\
Pulmonary artery pressure & $26.1 \pm 4.9$ & \\
$\quad$ (mm Hg) & & \\
MR & & \\
$\quad$ Moderate & & \\
$\quad$ Severe & &
\end{tabular}

$M R$, Mitral regurgitation; $H T N$, hypertension; $C O P D$, chronic obstructive pulmonary disease; $C R I$, chronic renal insufficiency; $B N P$, B-type natriuretic peptide; $N A$, not available; NYHA, New York Heart Association. *Number of patients, 9.

from end-diastole to end-systole at each intersection point within the myocardium (Figure 1). For registration, the user identified the posterior and anterior intersection points of the right ventricular free wall with the septum on the most basal short-axis image. Using these 2 points, and information from the LV geometry, the individual heart was registered to a standard 18-region finite element mesh (Figure 2). Within each element of the finite element mesh, displacements were fit in the least-squares sense to basis functions. The continuity of the displacement components was enforced at the element boundaries. The fitting of the basis functions to approximate the displacement data and calculation of the longitudinal and circumferential myocardial strain was performed using StressCheck (ESRD, Inc, St Louis, Mo).

\section{MRI-Based Multiparametric Strain Z-Score \\ Analysis}

A composite strain index was created using the circumferential and longitudinal strains, equally weighted, reflecting contractile function within the circumferential and longitudinal directions. Owing to the heterogeneous pattern of normal LV strain, the raw values were normalized to supply clinically relevant information. The method to relate individual patient-specific regional contractile function to a normal strain database (multiparametric strain analysis) has been previously described. ${ }^{12-16}$ In brief, z-scores generated from the raw strain values represent the number of standard deviations each raw value is from the mean of the group. The mean and standard deviations for both circumferential and longitudinal strain were calculated at each point of an encompassing grid of 15,300 LV points from the 60 normal volunteers. For comparison with this normal strain database, patient-specific $\mathrm{z}$-scores for both strain measures were calculated and averaged to obtain a multiparametric strain $\mathrm{Z}$-score representing the LV point-specific contractile function relative to normal. For each patient with MR, the z-scores were averaged over the 6 regions of the LV model (posteroseptal, anteroseptal, anterior, anterolateral, posterolateral, and posterior). 


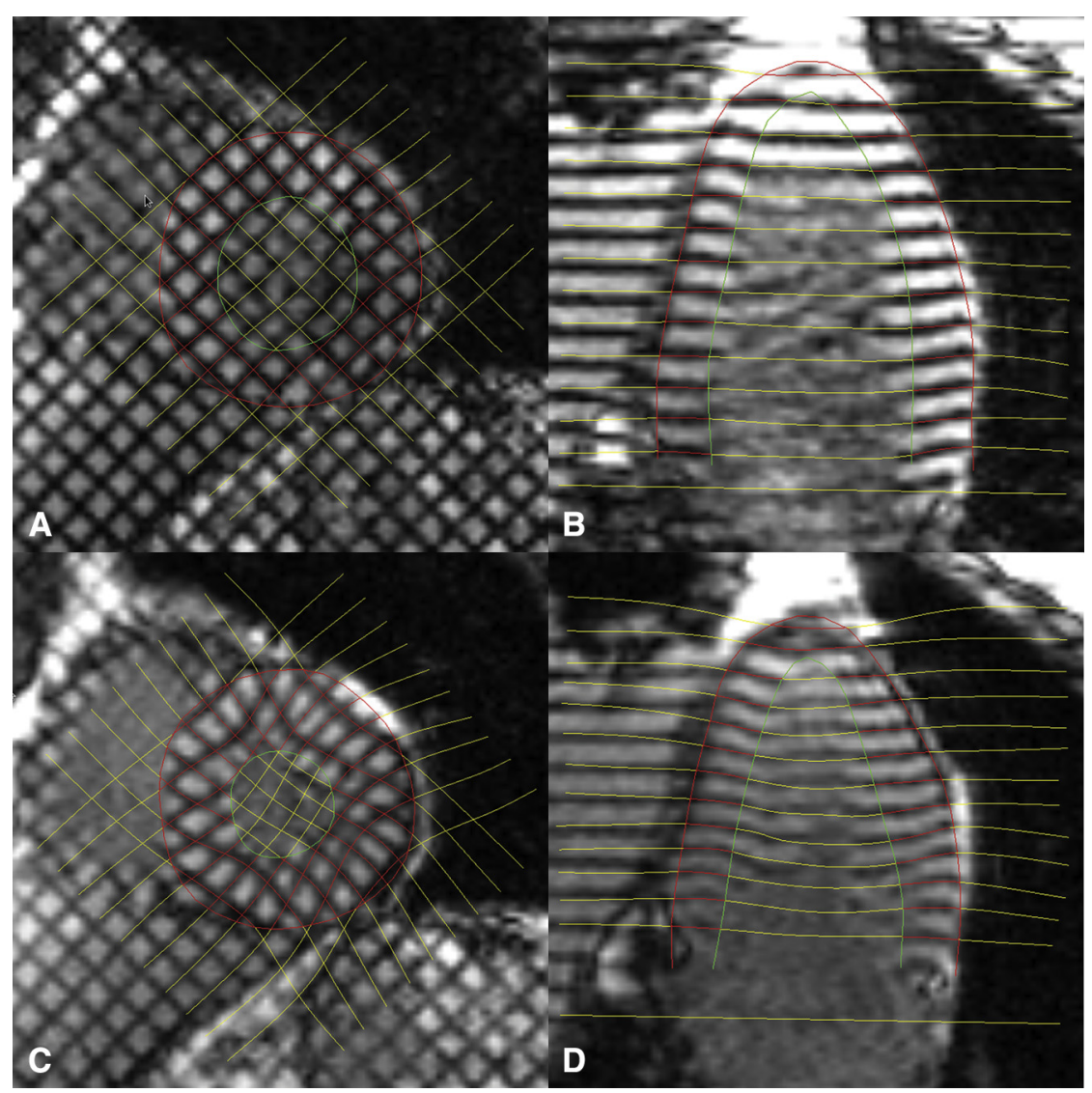

FIGURE 1. Images from (A and B) end-diastole and (C and D) end-systole, demonstrating deformations of tag lines during systole.

\section{Statistical Analysis}

Continuous data are presented as the mean \pm standard deviation and categorical data as percentages. Global strain comparisons between those with MR and the normal controls were performed using an independent samples $t$ test. Differences in the regional normalized contractile function among the patients with MR were assessed using a repeated measures analysis of variance. Sphericity of the data was assessed using Mauchly's test. The Bonferroni method was used for post hoc testing of the regional z-score data. All statistical tests were done using the statistical software package IBM SPSS 21 (IBM Corp, Armonk, NY).

\section{RESULTS}

\section{Echocardiographic Data for Patients With MR}

Using clinical echocardiography, the LV function, as assessed by the LV dimensions and ejection fraction $(\mathrm{EF})$, was within normal limits (Table 1) for this group of patients. The average left atrial size was increased compared with normal $(<3.8 \mathrm{~cm})$. The mean pulmonary arterial pressures for the entire group were either normal $(<25 \mathrm{~mm} \mathrm{Hg})$ or mildly elevated. MR was severe in $80 \%$ of the patients (Table 1 ). The mechanism of MR was posterior leaflet prolapse or flail posterior in $9(60 \%)$ of the studied patients. Five patients $(33 \%)$ had bileaflet prolapse, and $1(6 \%)$ had anterior prolapse.

\section{Global Strain for Patients With MR Versus Normal Controls}

LV function, as assessed by global myocardial strain and compared with that of the normal controls, is shown in Figure 3. Both circumferential strain $(-0.20 \pm$ 0.02 vs $-0.19 \pm 0.03)$ and longitudinal strain $(-0.16 \pm$ 0.03 vs $-0.15 \pm 0.03$ ) were greater in absolute value than those in the normal controls, but neither difference was significant $(P=.3$ and $P=.08$, respectively).

\section{Regional Multiparametric Strain}

Regional multiparametric z-scores were positive (least contractile) in the septal regions of the left ventricle and were maximally negative (most contractile) in the lateral walls (Figure 4). The standard deviations ranged from a low of 0.39 in the anteroseptal wall to a high of 0.71 in the anterior region. One-way repeated measures analysis of variance was applied to test for differences in the multiparametric z-scores among the 6 ventricular regions. The results of Mauchly's test indicated the assumption of sphericity was violated $\left[\chi^{2}(14)=33.51, P=.003\right]$. Thus, the degrees of freedom were adjusted using the GreenhouseGeisser estimate of sphericity $(\varepsilon=0.562)$. Overall, the 


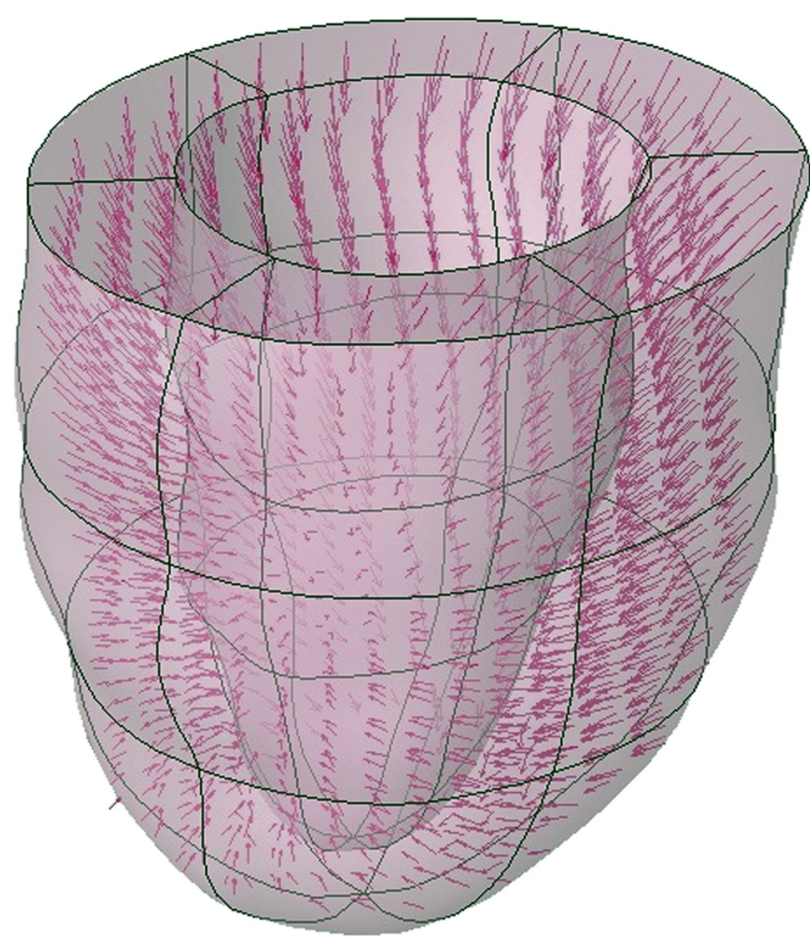

FIGURE 2. For each patient, the heart was registered to a standard 18-region finite element mesh, and displacements were computed at the intersection points of the tag surfaces. Model-predicted displacements and strains were computed from a least squares fitting of the measured displacement data.

multiparametric z-scores varied significantly by region $[\mathrm{F}(2.81,39.37)=7.21, P=.001]$. The anteroseptum was the least contractile region of the left ventricle and was significantly less contractile than the anterolateral $(P=.003)$, posterior $(P=.049)$, and posterolateral $(P=.009)$ walls. The posteroseptum was the next least contractile segment and was specifically less contractile than the anterolateral wall $(P=.035)$. The anterolateral segment was the most contractile of all segments, significantly greater than its anterior counterpart $(P=.002)$. Although the posterolateral walls also demonstrated greater contractility than the posterior segment, the difference was not statistically significant $(P=\mathrm{NS})$.

\section{DISCUSSION}

Multiple studies have addressed the limitations of traditional echocardiographic metrics in evaluating subclinical LV dysfunction among asymptomatic patients with MR. The inference, however, has been the incorporation of global strain indexes or neurohumoral markers in the evaluation of patients with MR to minimize the risk of developing permanent LV contractile injury might be intrinsically flawed. ${ }^{15,17-19}$ A principal finding of the present investigation was that in patients with severe degenerative MR, global contractile function, even when

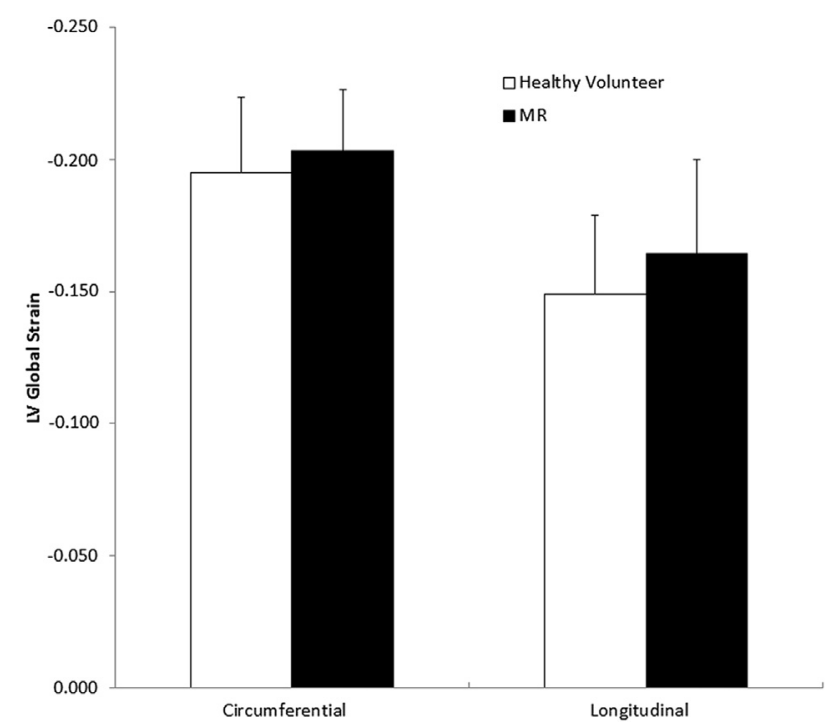

FIGURE 3. Global left ventricular function, as assessed by global myocardial circumferential and longitudinal strains, compared with normal controls. $L V$, Left ventricular; $M R$, mitral regurgitation.

assessed using multiparametric strain analysis and highresolution cardiac MRI tissue tagging, is preserved in the early stages of MR. This finding is not contradictory to the published data demonstrating decreased global strains before detectable changes in EF or chamber dilatation. However, it is more in agreement with studies demonstrating that, in minimally symptomatic patients with preserved EF, global strains might not only be easily preserved, but even hypercontractile compared with those of healthy volunteers. ${ }^{20,21}$ Similarly, although elevated BNP levels among patients with MR portend a worse prognosis, BNP, at its core, remains a neurohumoral marker of LV remodeling, suggesting that its elevation is unfortunately indicative of a maladaptive response that has already occurred within the left ventricle. ${ }^{17,19,22}$ The normal BNP levels and preserved global contractile function seen in our investigation suggest that the patients with MR in the present study were evaluated relatively early in the disease course and that the utility of global metrics in this setting could be limited.

The most important finding of the present investigation was that the earliest contractile injury seen in patients with degenerative MR and normal global contractile function is regionally and predictably distributed in the LV septum. These data further suggest that a compensatory increase in lateral wall contractility could exist, providing an explanation for the preserved global strain values demonstrated in the present series. To our knowledge, this is the first reported finding of such a regional contractile pattern demonstrated in patients with MR, in particular, when the overall contractile metrics are normal. These results build on data demonstrating regional contractile dysfunction 


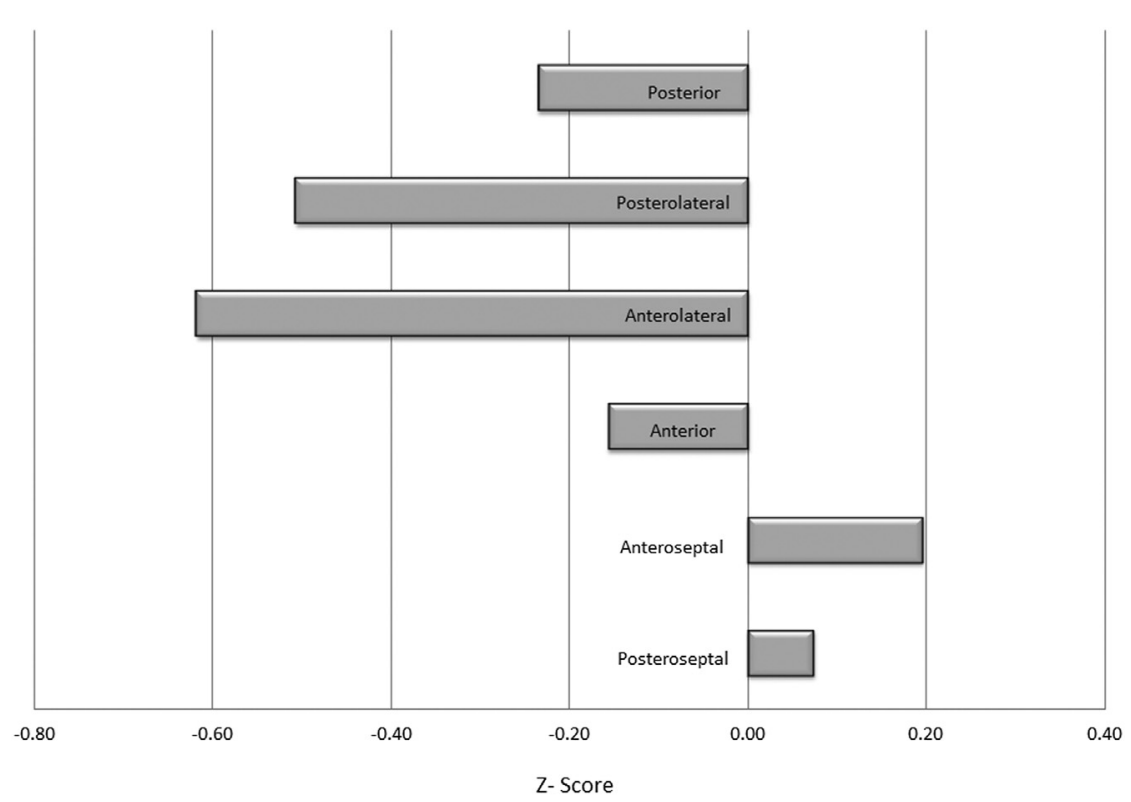

FIGURE 4. Regional ventricular function among patients with mitral regurgitation, assessed by multiparametric z-score analysis.

that has been observed in mitral valve prolapse or within the setting of congestive heart failure and dilated cardiomyopathy. ${ }^{15,23}$ This heterogeneous injury pattern is also consistent with experimental animal data suggesting that the earliest contractile injury in MR is not only likely to be regional, but might also vary across $\mathrm{LV}$ wall thickness. ${ }^{24}$

Although the ideal strain marker to detect subclinical LV dysfunction remains to be determined, the use of circumferential and longitudinal strains could be particularly appealing owing to their relative simplicity. These strain components are conceptually straightforward and, when focused on a specific anatomic region of interest (ie, LV septum) that is easily identifiable using both cardiac MRI and echocardiography, these metrics have particular appeal for both repeatability and subsequent adoption by healthcare providers. The finding of diminished contractile function along the LV septum, although not explained by the results of our investigation, is certainly hypothesis generating and might reflect regional heterogeneity in injury susceptibility or stress distribution.

The findings of the present study suggest that the EF might not be the ideal metric to determine surgical referral for asymptomatic patients with chronic MR. Patients' longterm outcomes have remained variable when surgical referral of asymptomatic patients has been based on traditional echocardiographic triggers, such as EF or chamber dilatation. ${ }^{18,20,22,25}$ Most critically, it has been shown that preserved preoperative EF does not ensure postoperative preservation of contractile function. ${ }^{26}$ Moreover, the regional heterogeneity of contractile injury confirmed in our study has highlighted the presence of as yet unidentified factors that induce nonuniform myocardial injury despite the global pathologic volume loading of MR on the left ventricle. The importance of using regional metrics of contractile function in assessing patients with MR is hereby suggested, because the septum appears reproducibly vulnerable and the lateral wall might well have an early compensatory role in maintaining global contractile function.

In an effort to preserve LV contractile function and optimize MV repair results, our findings suggest that it might be possible to track the earliest point of contractile injury among patients with MR using serial imaging and that subsequent investigational efforts should be geared toward identifying when regional septal dysfunction becomes either more widespread across the left ventricle or irreversible. ${ }^{26}$ Waiting for global decreases in myocardial strain is unlikely to be the ideal solution, because many patients with reduced global strains preoperatively will fail to have a normalized LV function postoperatively. ${ }^{21,22,27}$ However, with an improved understanding of the injury pattern and progression associated with MR, coupled with the use of more sophisticated metrics of regional contractile function, the preservation of $\mathrm{LV}$ function and subsequent improved patient survival after mitral valve surgery could become more standardized.

\section{Study Limitations}

The present study was limited by the MRI data acquisition process and the complexity of the data analysis that allowed only 15 patients with MR to contribute to the outcome analysis (60 normal volunteers contributed to the normal human strain database). Continued efforts to fully automate the data analysis could extend this investigative tool to larger patient populations. As an initial pilot study, 
the present investigation lacked a true longitudinal clinical comparison of the strain values of the patients over time. We are currently targeting patients both earlier in disease presentation and later in an attempt to more accurately define the contractile injury pattern by MR type (prolapse vs flail) and as MR evolves and progresses. The terms "asymptomatic" and "minimally symptomatic," although helpful, are clearly subjective and difficult to standardize. That the patients chosen for the present study demonstrated normal global function using echocardiography and multiparametric strain analysis and had normal BNP levels was reassured us that a relatively homogeneous group of patients with early MR was chosen for the present study.

\section{CONCLUSIONS}

Multiparametric strain analysis using high-resolution cardiac MRI and tissue tagging demonstrated a regional pattern of contractile injury in patients with minimally symptomatic and asymptomatic chronic MR that was not identifiable by any global contractile or biochemical marker. The LV septum appears to demonstrate contractile injury earliest in response to chronic MR, and compensatory mechanisms to maintain overall systolic function could include hypercontractile compensation by other regions. An alternative paradigm for triggering earlier surgical referral to preserve LV contractile function in patients with asymptomatic chronic MR should probably include the directed surveillance of early injury (or "sentinel") LV regions (LV septum) using high-resolution metrics of regional contractile function.

\section{References}

1. Nkomo VT, Gardin JM, Skelton TN, Gottdiener JS, Scott CG, EnriquezSarano M. Burden of valvular heart diseases: a population-based study. Lancet. 2006;368:1005-11.

2. Enriquez-Sarano M, Avierinos JF, Messika-Zeitoun D, Detaint D, Capps M, Nkomo V, et al. Quantitative determinants of the outcome of asymptomatic mitral regurgitation. N Engl J Med. 2005;352:875-83.

3. Ling LH, Enriquez-Sarano M, Seward JB, Tajik AJ, Schaff HV, Bailey KR, et al. Clinical outcome of mitral regurgitation due to flail leaflet. N Engl J Med. 1996; 335:1417-23

4. David TE, Ivanov J, Armstrong S, Christie D, Rakowski H. A comparison of outcomes of mitral valve repair for degenerative disease with posterior, anterior, and bileaflet prolapse. J Thorac Cardiovasc Surg. 2005;130:1242-9.

5. Gillinov AM, Blackstone EH, Nowicki ER, Slisatkorn W, Al-Dossari G, Johnston DR, et al. Valve repair versus valve replacement for degenerative mitral valve disease. J Thorac Cardiovasc Surg. 2008;135:885-93.e1-2.

6. Gillinov AM, Mihaljevic T, Blackstone EH, George K, Svensson LG, Nowicki ER, et al. Should patients with severe degenerative mitral regurgitation delay surgery until symptoms develop? Ann Thorac Surg. 2010;90:481-8.

7. Bonow RO, Carabello BA, Chatterjee K, de Leon AC Jr, Faxon DP, Freed MD, et al. ACC/AHA 2006 guidelines for the management of patients with valvular heart disease: a report of the American College of Cardiology/American Heart Association Task Force on Practice Guidelines (writing Committee to Revise the 1998 guidelines for the management of patients with valvular heart disease) developed in collaboration with the Society of Cardiovascular Anesthesiologists endorsed by the Society for Cardiovascular Angiography and Interventions and the Society of Thoracic Surgeons. J Am Coll Cardiol. 2006;48:e1-148.

8. Topilsky Y, Suri R, Schaff HV, Enriquez-Sarano M. When to intervene for asymptomatic mitral valve regurgitation. Semin Thorac Cardiovasc Surg. 2010;22:216-24

9. Axel L, Dougherty L. Heart wall motion: improved method of spatial modulation of magnetization for MR imaging. Radiology. 1989;172:349-50.

10. Moulton MJ, Creswell LL, Downing SW, Actis RL, Szabo BA, Vannier MW, et al. Spline surface interpolation for calculating 3-D ventricular strains from MRI tissue tagging. Am J Physiol. 1996;270:H281-97.

11. Moustakidis P, Cupps BP, Pomerantz BJ, Scheri RP, Maniar HS, Kates AM, et al Noninvasive, quantitative assessment of left ventricular function in ischemic cardiomyopathy. J Surg Res. 2004;116:187-96.

12. Brady BD, Knutsen AK, Ma N, Gardner R, Taggar AK, Cupps BP, et al. MRIbased multiparametric strain analysis predicts contractile recovery after aortic valve replacement for aortic insufficiency. J Card Surg. 2012;27:415-22.

13. Cupps BP, Bree DR, Wollmuth JR, Howells AC, Voeller RK, Rogers JG, et al. Myocardial viability mapping by magnetic resonance-based multiparametric systolic strain analysis. Ann Thorac Surg. 2008;86:1546-53.

14. Cupps BP, Taggar AK, Reynolds LM, Lawton JS, Pasque MK. Regional myocardial contractile function: multiparametric strain mapping. Interact Cardiovasc Thorac Surg. 2010;10:953-7.

15. Joseph S, Moazami N, Cupps BP, Howells A, Craddock H, Ewald G, et al. Mag netic resonance imaging-based multiparametric systolic strain analysis and regional contractile heterogeneity in patients with dilated cardiomyopathy. $J$ Heart Lung Transplant. 2009;28:388-94.

16. Knutsen AK, Ma N, Taggar AK, Brady BD, Cupps BP, Pasque MK. Heterogeneous distribution of left ventricular contractile injury in chronic aortic insufficiency. Ann Thorac Surg. 2012;93:1121-7.

17. Detaint D, Messika-Zeitoun D, Avierinos JF, Scott C, Chen H, Burnett JC Jr, et al B-type natriuretic peptide in organic mitral regurgitation: determinants and impact on outcome. Circulation. 2005;111:2391-7.

18. Mankad R, McCreery C, Rogers WJ, Weichmann RJ, Savage E, Reichek N, et al Regional myocardial strain before and after mitral valve repair for severe mitral regurgitation. J Cardiovasc Magn Reson. 2001;3:257-66.

19. Pizarro R, Bazzino OO, Oberti PF, Falconi M, Achilli F, Arias A, et al. Prospective validation of the prognostic usefulness of brain natriuretic peptide in asymptomatic patients with chronic severe mitral regurgitation. J Am Coll Cardiol. 2009;54:1099-106.

20. Witkowski TG, Thomas JD, Delgado V, van Rijnsoever E, Ng AC, Hoke U, et al Changes in left ventricular function after mitral valve repair for severe organic mitral regurgitation. Ann Thorac Surg. 2012;93:754-60.

21. Zito C, Carerj S, Todaro MC, Cusma-Piccione M, Caprino A, Di Bella G, et al. Myocardial deformation and rotational profiles in mitral valve prolapse. Am J Cardiol. 2013;112:984-90.

22. Hwang IC, Kim YJ, Kim KH, Lee SP, Kim HK, Sohn DW, et al. Prognostic value of B-type natriuretic peptide in patients with chronic mitral regurgitation undergoing surgery: mid-term follow-up results. Eur J Cardiothorac Surg. 2013;43: e1-6.

23. Malev E, Zemtsovsky E, Pshepiy A, Timofeev E, Reeva S, Prokudina M. Evaluation of left ventricular systolic function in young adults with mitral valve prolapse. Exp Clin Cardiol. 2012;17:165-8.

24. Carlhall CJ, Nguyen TC, Itoh A, Ennis DB, Bothe W, Liang D, et al. Alterations in transmural myocardial strain: an early marker of left ventricular dysfunction in mitral regurgitation? Circulation. 2008;118:S256-62.

25. Enriquez-Sarano M, Sundt TM III. Early surgery is recommended for mitral regurgitation. Circulation. 2010;121:804-11; discussion 812.

26. Marciniak A, Sutherland GR, Marciniak M, Kourliouros A, Bijnens B, Jahangiri M. Prediction of postoperative left ventricular systolic function in patients with chronic mitral regurgitation undergoing valve surgery-the role of deformation imaging. Eur J Cardiothorac Surg. 2011;40:1131-7.

27. Pomerantz BJ, Wollmuth JR, Krock MD, Cupps BP, Moustakidis P, Kouchoukos NT, et al. Myocardial systolic strain is decreased after aortic valve replacement in patients with aortic insufficiency. Ann Thorac Surg. 2005;80: 2186-92. 